\title{
Dermal leishmaniasis in a 25-year-old Syrian refugee
}

\author{
Scott Bradshaw MD, Ivan V. Litvinov MD PhD
}

— Cite as: CMAJ 2017 November 13;189:E1397. doi: 10.1503/cmaj.170844

A n otherwise healthy 25-year-old Syrian man arrived in Canada as part of the country's Syrian refugee program. The patient presented to our dermatology clinic with nonresolving indurated plaques on his forehead and left hand (Figures $1 \mathrm{~A}$ and 1B). The plaques had been present for four weeks and were otherwise asymptomatic. For our differential diagnosis, we used the "Five L's" for red dermal plaques on the face: lupus erythematosus/tumid lupus, Jessner lymphocytic infiltrate, lymphoma cutis, lymphocytoma cutis and polymorphous light eruption. ${ }^{1,2}$ Because of our patient's country of origin, we also considered leprosy and leishmania. Great imitators, syphilis (lues) and sarcoid, can also be considered in this setting. A skin biopsy of the patient's forehead plaque showed a dense lymphocytic and histiocytic infiltrate in the superficial and mid dermis. The histiocytes showed intracellular organisms with kinetoplasts, but no capsule on the Giemsa and hematoxylin and eosin stains (Figures $1 \mathrm{C}$ and 1D. Additional images are available in Appendix 1, at www.cmaj.ca/lookup/suppl/doi:10.1503/cmaj $.170844 /-/ D C 1)$. Amastigotes were arranged radially around the periphery of the histiocyte. Polymerase chain reaction testing for leishmania identified the Leishmania tropica species of the parasite.

We referred our patient to the tropical disease clinic and after considering the cost versus benefit of intralesional sodium stibogluconate (not covered under the provincial drug benefit formulary), our patient chose treatment with three courses of cryotherapy, ${ }^{3}$ which led to a complete resolution of the lesions. Overall, when selecting a treatment, it is important to consider the specific strain of Leishmania and the extent of skin and systemic involvement. 4,5 Pentavalent antimonial compounds, amphotericin B and other systemic treatments can be used in cases where extensive skin or systemic disease is confirmed. ${ }^{4,5}$

\section{References}

1. Dermatology notes: the five L's. Hollywood (FL): Minars Dermatology; 2012. Available: www.minarsdermatology.com/dermatology_textbook/index.html?dt_the_ five_ls.htm (accessed 2017 Mar. 13).

2. Dermatopathology and basic science: superficial and deep perivascular infiltrates 5 L's. Torrance (CA): Dermatopathology Institute; 2017. Available: www.

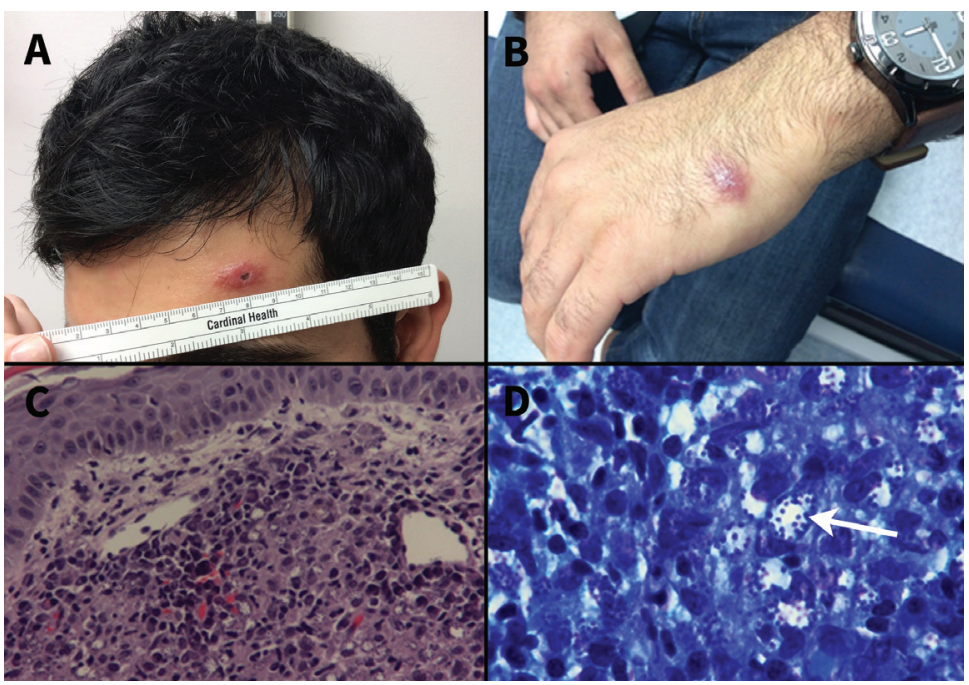

Figure 1: (A) Indurated erythematous plaque on the left side of the forehead of a 25-year-old man, with a biopsy scar in the centre of a lesion. (B) Indurated solitary plaque on the patient's left hand. (C) Hematoxylin and eosin staining of the forehead skin biopsy at $40 \mathrm{x}$ magnification, showing a dense lymphocytic and histiocytic infiltrate in the superficial and mid dermis. (D) Giemsa staining highlights leishmania amastigotes arranged radially within the histiocyte (arrow).

dermpathmd.com/mnemonics/mnemonics_dermatopathology.htm (accessed 2017 Mar. 17).

3. Monge-Maillo B, Lopez-Velez R. Therapeutic options for old world cutaneous leishmaniasis and new world cutaneous and mucocutaneous leishmaniasis. Drugs 2013;73:1889-920.

4. Demers E, Forrest DM, Weichert GE. Cutaneous leishmaniasis in a returning traveller. CMAJ 2013;185:681-3.

5. Wolf Nassif P, DE Mello TFP, Navasconi TR, et al. Safety and efficacy of current alternatives in the topical treatment of cutaneous leishmaniasis: a systematic review. Parasitology 2017;144:995-1004.

\section{Competing interests: None declared.}

This article has been peer reviewed.

The authors have obtained patient consent.

Affiliations: Department of Pathology (Bradshaw); Division of Dermatology (Litvinov), University of Ottawa, Ottawa, Ont.

Correspondence to: Ivan Litvinov, ivan.litvinov@mcgill.ca 